Activités

$18-2$ | 2021

Espace et temps de l'activité / Travail, Territoire et politique

\title{
Espace, Travail et numérique. Le cas du travail en flex office
}

Space, work and digital transformations. The case of work in flex office

\section{Nadia Heddad}

\section{(2) OpenEdition}

\section{Journals}

Édition électronique

URL : https://journals.openedition.org/activites/6605

DOI : 10.4000/activites.6605

ISSN : 1765-2723

Éditeur

ARPACT - Association Recherches et Pratiques sur les ACTivités

Référence électronique

Nadia Heddad, "Espace, Travail et numérique. Le cas du travail en flex office », Activités [En ligne],

18-2 | 2021, mis en ligne le 15 octobre 2021, consulté le 07 avril 2022. URL : http://

journals.openedition.org/activites/6605; DOI : https://doi.org/10.4000/activites.6605

Ce document a été généré automatiquement le 7 avril 2022.

\section{cc) (i) $९$}

Activités est mis à disposition selon les termes de la licence Creative Commons Attribution - Pas d'Utilisation Commerciale - Pas de Modification 4.0 International 


\title{
Espace, Travail et numérique. Le cas du travail en flex office
}

Space, work and digital transformations. The case of work in flex office

\author{
Nadia Heddad
}

\section{NOTE DE L'ÉDITEUR}

Article soumis le 07/09/2020, accepté le 28/05/2021

\section{Introduction}

1 Toute activité humaine s'inscrit dans un espace. Cet espace est pour partie matériel, lié à l'environnement physique qui nous entoure, mais il est aussi organisationnel et relationnel. Rapporté aux lieux dédiés au travail, l'espace est non seulement structuré par ceux qui l'ont conçu. Il est ré-agencé et organisé par ceux qui y travaillent. D'un côté, l'espace attribué par l'organisation du travail structure l'action en lui offrant un support ou en la contraignant. De l'autre, l'espace est structuré par l'activité de travail qui s'y déploie. Il est agi par l'activité de travail qui y prend place.

2 Le développement des techniques participe depuis toujours à l'évolution des activités humaines, mais le déploiement massif de l'usage des outils numériques dans les organisations de travail est contemporain. Il modifie profondément le rapport à l'activité de travail, mais aussi à l'espace.

3 Ce texte propose de questionner en quoi l'évolution du numérique dans les organisations du travail modifie l'espace, notamment dans le cadre du modèle du flex office. L'analyse s'ancre dans le champ de l'ergonomie de l'activité et cherche à aborder la relation entre activité de travail et espace. Elle vise à interroger les perspectives du point de vue du travail. 
Les propos sont structurés autour de six parties successives :

1. La question de la spécificité des espaces dans les organisations du travail. Il s'agit de distinguer en quoi les espaces dédiés au travail se démarquent des autres lieux de vie, notamment de l'espace de l'habitat ou de la ville.

2. La question spatiale dans le travail du point de vue de la tâche et de l'activité de travail. Une approche théorique est ici proposée pour comprendre l'activité de façon conjointe à l'espace de l'activité de travail. Elle distingue l'approche par la tâche dans la conception de l'approche par l'activité de travail qui ne se comprend que dans le rapport intime au déploiement du travail qui, ce faisant, transforme les espaces conçus.

3. La question de l'introduction massive du numérique dans les espaces dédiés au travail. Le développement du numérique et l'évolution des espaces dans les entreprises sont en effet reliés. Les deux conduisent à modifier les politiques immobilières des entreprises du secteur tertiaire à partir d'un nouveau modèle d'organisation de l'espace, celui du flex office.

4. La présentation de l'étude des cas et des méthodes. Il s'agit d'une entreprise française de télécommunications qui a fait le choix d'introduire le modèle d'organisation spatiale en flex office. La demande vise un retour d'expérience un an et demi après la conception des aménagements. Les méthodes utilisées s'appuient sur l'analyse de l'activité développée en ergonomie, avec un focus fort sur la dimension spatiale du travail.

5. La présentation des résultats de l'analyse. Ils interrogent la place du travail dans le cadre du modèle reliant numérique et espace, le flex office. Cette partie aborde les caractéristiques du modèle flex office. Sont identifiées au total, quatre spécificités. Les deux premières questionnent la manière dont l'espace, prévu par les concepteurs, participe de la tâche. Les deux autres montrent la manière dont l'activité de travail se déploie. D'un côté, le travail se réalise dans une forme de porosité spatiale et temporelle dans des espaces au sein et en dehors de l'entreprise. De l'autre, le travail en flex office renforce le développement du numérique qui devient à la fois une condition et une résultante pour le travail dans une espace en flex office.

6. La discussion. Elle ouvre des perspectives du point de vue du travail en pointant la question des effets du développement imbriqué du numérique et de l'espace dans le modèle du flex office. Elle propose quelques pistes conceptuelles et méthodologiques pour saisir la question du travail dans ce contexte de forte évolution numérique et spatiale.

5 En abordant l'évolution du numérique et des espaces dans le tertiaire, autour de la figure du modèle du flex office, ce texte invite à revisiter la notion de situation de travail en l'enrichissant de la dimension spatiale. Il est proposé d'approcher la question du travail dans une double perspective : d'un côté, la manière dont la conception considère le travail, au travers des critères mis en avant par les concepteurs, c'est-à-dire l'espace de la tâche, et de l'autre, la façon dont le travail conduit à modifier l'espace en faisant émerger dans la dynamique de l'activité de travail un autre rapport à l'espace. Il s'agit de l'espace propre à l'activité qui redéfinit une autre unité que celle envisagée par le découpage des locaux, zones et postes.

\section{La spécificité des espaces dans les organisations de travail}

6 Contrairement à l'espace de l'habitat, investi comme sujet d'étude pour comprendre la relation entre l'espace et les habitants, peu d'études concernent les espaces dédiés au travail, notamment sous l'angle de l'activité de travail réel. Fischer $(1978,2016)$ explore le concept d'espace de travail en milieu industriel et apporte un regard psychologique 
comportemental sur le lien entre les individus et les aménagements d'espace de travail. Goffman $(1973,1974)$ investit les relations qui se forment entre les individus sous l'angle sociologique. Il s'appuie sur des métaphores empruntées au théâtre en explorant les espaces archétypes comme les salles de jeux ou les lieux de rencontre. Il analyse l'espace comme un système de places où les individus rentrent en relation selon un rituel et une figuration dans une logique de valorisation de chacun. L'espace est, pour lui, le support pour des relations situées. Hall (1966/1971) vient instruire et enrichir la dimension culturelle dans les usages de l'espace en montrant notamment comment la distance entre deux personnes participe de la communication. Il donne une lecture culturelle aux comportements des individus dans la manière de se tenir dans l'espace'.

7 Les espaces dédiés au travail présentent une double spécificité. La première est qu'il s'agit de lieux où se manifeste le lien de subordination. Dans l'organisation des entreprises, toute personne est engagée dans une relation hiérarchique qui la relie à d'autres dans un rapport précis à la décision. Ce rapport de subordination n'est pas neutre. Les équipes engagées dans la production sont encadrées et évaluées sur des résultats à atteindre sur la base d'un travail prescrit. La seconde spécificité est qu'il s'agit d'organisations hétéronomes (Cazamian, 1996). Ceux qui pensent les moyens, notamment en matière d'espace, ne sont pas ceux qui les pratiquent au quotidien. Le bâti, les locaux, les aménagements, les postes et les équipements font partie du cadre matériel spatial mis à la disposition du travail par l'organisation.

Le vocable espace demeure cependant flou (Cazamian \& Lautier, 1996 ; Heddad, 2017a ; Lautier, 1999). Il ne désigne pas nécessairement les mêmes choses selon que l'on soit du côté des acteurs de la conception et de la décision ou du côté de ceux qui vivent l'espace au quotidien dans la réalisation de l'activité de travail.

Du côté des concepteurs et des décideurs, l'espace constitue un socle qu'il convient de modeler pour le rendre conforme à l'organisation du travail visée. Il doit contenir l'ensemble des moyens humains et matériels pour réaliser la production qu'elle soit industrielle ou de service. L'accent est mis sur la recherche d'un modèle d'espace pour soutenir une visée organisationnelle qui se traduit dans l'espace. Un déménagement, un réaménagement ou la construction d'un nouvel édifice est de ce point de vue bien souvent l'occasion de modifier l'organisation. Une organisation spatiale vient soutenir une organisation du travail qui doit orienter les pratiques des lieux de ceux qui y travaillent. Dans cette approche, le modèle spatial s'inspire d'un idéal à mettre en avant pour changer les pratiques spatiales. Cette idée rejoint celle d'un idéal transcendantal avec un modèle à atteindre (Kant, 1781). Dans cette approche, l'accent est fortement mis sur l'artefact spatial comme un a priori, un objet d'attention soutenant des intentions organisationnelles visées par les concepteurs et les décideurs. Les discours managériaux viennent ensuite compléter cette conception d'un idéal devant orienter les pratiques spatiales.

10 Du côté de ceux qui pratiquent les lieux, l'espace est un support sur lequel ils peuvent s'appuyer, ou non, pour accomplir l'activité de travail. L'espace est alors soit une contrainte, soit une ressource pour réaliser le travail (Camus, Évette, \& Fabre 1991; Falzon, 2013 ; Fenker, 2003 ; Goffman, 1974 ; Heddad, 2017a, 2017b ; Kirsh 1995 ; Lautier, 1999, 2001).

11 En tant que sujet agissant, l'agent, l'opérateur ou le collaborateur fait l'expérience incarnée d'un espace qui lui est proposé pour la réalisation de son activité de travail. Il 
peut le transformer, mais aussi modifier sa disposition pour l'ajuster à son travail. Ces évolutions s'inscrivent dans un temps long qualitatif (Gaudart, 2014) relié à ceux qui pratiquent et habitent les lieux dans la durée. Cette idée rejoint celle développée par Poincaré (1902) pour qui l'espace est avant tout une expérience. En soutenant l'idée que l'espace est le résultat d'une expérience et non d'un idéal qui précèderait toute sensibilité, il s'oppose explicitement à Kant.

En conception, les espaces de travail sont bien souvent considérés comme cet a priori à toute sensibilité qu'il faut imaginer pour bien mener un projet spatial. L'espace comme une expérience est une démarche plus rarement investie dans les organisations de travail. En effet, le retour d'expérience à partir des points de vue de ceux qui travaillent au sein des lieux de travail, ceux pour qui la conception est dédiée, n'est pas une pratique des acteurs du milieu de la conception des espaces dans le monde du travail. L'évaluation des projets n'intègre que trop rarement des démarches qui invitent les équipes qui vivent au quotidien les espaces. L'évaluation dans le milieu du bâtiment s'opère généralement entre pairs, c'est-à-dire entre experts du bâti dont la représentation est homogène aux métiers du bâtiment. Elle n'intègre pas la question de l'expérience spatiale des « habitants » des lieux. Cette situation est spécifiquement plus marquée dans le cas des lieux de travail.

13 En effet, dans l'habitat, les pratiques de programmation urbaine et de conception évoluent peu à peu vers une pratique plus ouverte à la possible participation des citoyens à la fabrication de la ville et la conception des grands équipements publics (Zetlaoui-Léger, 2013) ou des personnes âgées (Couillaud, 2018).

14 En revanche, pour les lieux de travail, les pratiques des acteurs décisionnels de la conception ont peu évolué. Pourtant, l'intégration d'ergonomes à la conception d'espace de travail est une pratique qui existe depuis les années 80 (Dejean, Pretto, \& Renouard, 1988). Elle a permis de développer en ergonomie une démarche d'intervention en conception architecturale (Martin, 2000) s'inspirant des approches développées pour l'intervention en milieu industriel (Daniellou, 1988, 2004 ; Daniellou \& Garrigou, 1993).

15 Le milieu de la conception d'espace de travail demeure malgré tout encore imprégné de méthodes intégrant difficilement la question du travail.

\section{La question spatiale dans le travail}

Cela a été évoqué, toute situation de travail comprend une dimension spatiale. Elle est relative au corps, car le rapport à l'espace est incarné. La question de l'échelle et de l'orientation du corps dans l'espace se pose, mais pas seulement.

17 L'activité de travail se réalise dans une unité relative à l'échelle humaine. Il s'agit d'un repère qui a du sens pour celle ou celui qui déploie et vit son activité. Du côté des concepteurs ou des décideurs, cette question de l'échelle peut être enjambée au bénéfice d'une vue d'ensemble du système technique et organisationnel. Un plan sur un format de présentation (papier ou sur un écran) précisant un aménagement indiquant les différents locaux ou zones ne donne pas nécessairement accès à ce que cela peut représenter au niveau d'un agent à son poste de travail. De plus, le corps humain se caractérise par le fait qu'il est orienté. La structure corporelle, au travers de la disposition de la tête au-dessus de la colonne vertébrale, l'emplacement des yeux sur la 
face avant de la tête, l'orientation des membres supérieurs et inférieurs, participe de la manière dont tout le corps peut se mouvoir dans l'espace.

Un ancrage spatial du corps est ainsi nécessaire pour le déploiement de l'activité et cela même si les relations peuvent passer par des systèmes qui s'affranchissent d'une présence physique. Le recours à des technologies de la communication modifie ainsi le rapport à l'espace, mais ne le supprime pas. Il l'étend au-delà de la présence physique dans une lecture spatiale plus complexe. Toute activité est inscrite dans un espace présentant des propriétés matérielles et relationnelles : les sujets échangent et agissent entre eux, mais aussi avec les objets qui les entourent pour réaliser l'activité de travail.

En ergonomie, la pratique de l'analyse de l'activité de travail permet d'éclairer les exigences du travail et ce faisant, mettre l'accent sur les critères à prendre en compte en conception. Il est ici proposé d'enrichir cette analyse de l'activité par une analyse spatiale qui permet de l'étayer par une compréhension fine du rapport entre le déroulé de l'activité de travail et l'espace dans lequel le travail prend place. Cette lecture est double.

La première est le rapport entre l'espace et la tâche. L'espace, pensé comme un contenu, une surface ou un agencement, est partie constituante de la tâche. Dans le même temps, les personnels ne sont jamais neutres dans la manière d'investir un espace qui leur est attribué. Ils modifient et transforment les agencements que ce soit des locaux, des matériels ou des équipements qui leur sont affectés. Ils ajustent leur espace.

21 La seconde lecture est relative à cette dimension incarnée de toute activité humaine dans un espace. L'activité est située. Elle prend, au travers du corps du sujet agissant, appui sur un espace pour constituer une unité propre à l'activité de travail. Dans cette perspective, l'analyse spatiale appliquée à l'analyse de l'activité vient enrichir la notion de situation de travail mise en lumière en analyse de l'activité de travail.

Cette relecture de l'activité par l'espace montre que le travail déborde et remodèle le découpage spatial pensé par l'organisation du travail qui raisonne bien souvent par séparation (des surfaces, des locaux, des zones ou des postes de travail). Il s'agit ici de l'espace propre à l'activité qui ne peut se comprendre que de façon endogène à l'activité de travail. Dit autrement, toute intervention visant la transformation des situations de travail peut s'enrichir d'une lecture spatiale du travail en analyse de l'activité pratiquée en ergonomie.

\subsection{L'espace, une dimension de la tâche, mais aussi un résultat de l'activité}

La spécificité de l'espace est qu'il détermine tout aussi bien les conditions dans lesquelles s'opère une activité de travail, tout comme il en est le résultat (Cazamian \& Lautier 1996; Heddad, 2017 ; Lautier 1999). L'espace propre à l'activité est à la fois un support et un produit de l'activité de travail dont il ne peut être séparé (Heddad, 2017a, 2017b). Il est possible d'approcher l'espace sous différents angles dans les organisations du travail.

- Le premier est le plus courant et consiste à aborder l'espace par le poste, les aménagements, les équipements, la superficie et les locaux. Il s'agit de l'espace relatif à la tâche. 
- Le deuxième propose d'approcher l'espace comme un support qui peut être une ressource ou une contrainte pour l'activité. Il est en lien avec le travail réel.

- Le troisième axe consiste à approcher l'espace comme étant à la fois le dispositif matériel, organisationnel et relationnel, support et produit des relations, des coordinations, des coopérations et moyen de toutes les formes de communication verbale ou non. Il s'agit de la double relation qu'engendre le déploiement de toute activité dans un espace dans lequel elle prend place.

Chacun de ces axes est développé ci-dessous.

\subsection{L'espace de la tâche}

Dans les organisations du travail, l'espace mis à la disposition des équipes conditionne et influe le déroulement des activités de travail. Il est un support offert pour la réalisation du travail attendu. L'espace conçu, au sens de Lefebvre (1974/2000), c'est à dire tel qu'il a été pensé par les concepteurs, participe de la prescription du travail. Il relève du registre de la tâche et correspond à l'espace limité au poste de travail ou au local dans lequel il a été envisagé par l'organisation du travail. Il relève également des ambiances physiques prévues par les concepteurs. Parmi elles, la qualité des lieux sur plan de l'éclairage, de l'acoustique, des conditions thermiques, etc.

Proposer un agencement de l'espace au travers d'une organisation spatiale spécifique induite par le bâti ou par l'aménagement intérieur, témoigne d'une volonté d'agir sur le déroulé du travail des équipes. Faire tomber les cloisons, regrouper les équipes dans un même local sont parmi les figures classiques d'action sur les espaces dans une visée de transformation de l'organisation. Recourir aux technologies pour les réunir ou les coordonner procède de la même logique prescriptive. Une organisation spatiale (forme organisationnelle, proximité ou distanciation physique et relationnelle) peut venir appuyer une activité de travail comme elle peut la contraindre.

Ce découpage à partir de la tâche guide les concepteurs, notamment pour penser une organisation spatiale qui se traduit par un aménagement d'espace ou un édifice. C'est cette manière de diviser l'espace qui est valorisée lors des projets de transformation. Elle constitue une grille de lecture simplifiée tant de l'activité de travail que des espaces dédiés au travail. La dimension matérielle (technique) rapportée aux différents postes et les locaux de travail est mise en avant. Elle est décrite et transmise aux concepteurs qui s'en inspirent pour penser de nouveaux espaces. Ils reproduisent à leur tour ce découpage qui raisonne par séparation des locaux, des zones ou des postes de travail. C'est une logique qui opère par la rationalisation des séparations de l'ordre de la tâche. Elle contribue aujourd'hui à structurer la rédaction des cahiers des charges, nommés programmes architecturaux, dans les projets de bâtiment.

L'espace de la tâche correspond ainsi à un assemblage de moyens matériels et organisationnels dédiés au travail de production à partir du point de vue des acteurs de l'organisation du travail.

\subsection{L'espace, résultat de l'activité}

Les équipes qui investissent les espaces en situation d'activité de travail quant à elles les vivent comme une dimension faisant partie intégrante de la situation de travail. Elles modifient et agissent sur l'artefact spatial. Il s'agit de l'espace ajusté par et dans le 
travail (Heddad, 2017a ; Lautier, 1999) des personnels qui le modifient et l'adaptent pour répondre aux objectifs de production qui leur sont assignés.

Ils déplacent les équipements et les aménagements mis à leur disposition. Ils (ré)agencent les mobiliers, peuvent obstruer une cloison vitrée ou une fenêtre en y apposant des affiches, un calendrier mural ou des photos. Ils peuvent aussi utiliser des accès et des circulations autres que ceux prévus. Ils opèrent des réaffectations fonctionnelles des locaux en transformant des espaces de circulation en lieux de stockage, des coins café sont improvisés dans les espaces de travail, des portes prévues pour répondre aux contraintes de la sécurité incendie peuvent être utilisées comme raccourci, des poteaux ou des cloisons sont utilisés pour appuyer du matériel roulant, etc.

31 Les traces de l'activité peuvent se lire dans les espaces. Des murs abimés portant les marques de chariots ou brancards, des sols usés par les nombreux piétinements devant un ascenseur ou encore des portes ou parois gondolées d'un monte-charge sont des traces de l'activité. Ce sont des marqueurs de la pratique des espaces dans un temps pas toujours considéré, celui du temps qualitatif de l'expérience des lieux en référence au temps long de Gaudart (2014) appliqué au vécu des équipes de travail dans un édifice. Le bâti, en vieillissant et se confrontant dans la durée aux pratiques spatiales des professionnels, laisse entrevoir les traces des activités. Il est marqué dans le temps par les pratiques spatiales de ses occupants.

\subsection{L'espace de l'activité, support et produit de l'activité}

Du point de vue de ceux qui réalisent une activité de travail, l'espace n'est pas un simple assemblage matériel et organisationnel. Il est un système, un tout globalisant qui fait sens pour ceux qui y travaillent. Il ne se comprend qu'à partir du travail réel, de façon endogène à l'activité dans la manière dont elle se met en œuvre concrètement par ceux qui réalisent le travail. Cette idée rejoint la perspective développée par la phénoménologie de Merleau-Ponty (1945) pour qui l'espace ne se comprend qu'à l'intérieur du champ perceptif du sujet agissant.

Cet espace est concret pour ceux qui y travaillent. Les dynamiques d'agir et le caractère corporel et incarné de toute activité réalisée dans un lieu sont à considérer dans un même temps.

En tant que «production locale » (Suchman, 1996), cet espace est émergeant de l'agir. L'activité de travail se réalise dans un continuum au sens de Varela, Thompson et Rosch (1993) imbriquant dimension spatiale et déroulé de l'activité dans un processus qui émane de l'agir comme expérience du sujet au monde. L'activité de travail et la manière dont celle-ci se traduit spatialement (en s'appuyant et façonnant l'espace) ne font qu'un. Les deux sont intimement reliées et font système pour ceux qui vivent dans la durée et de façon incarnée, le déploiement de leur activité de travail dans un lieu précis.

Pour saisir cette dimension spécifique de l'espace relié au travail réel, l'espace de l'activité de travail (Heddad, 2017b) propose une unité d'analyse associant deux registres:

1. L'espace physique et organisationnel, relevant de la tâche, mais ajusté et modifié dans et par le travail. Il comprend tant le bâti que les aménagements et les équipements associés (Figure 1) mis à la disposition des personnels. Cet espace est transformé par et dans la 
réalisation de l'activité de travail qui le modèle (Figure 2). La prise d'information s'opère au travers de la manière d'occuper l'espace, mais aussi de la façon dont chacun se tient et se meut dans l'espace. Prendre connaissance de la posture d'un collègue en train de réaliser une tâche donne accès à une compréhension de ce qui se joue dans la situation de travail du collègue et permet d'anticiper les effets sur le travail de chacun dans le collectif (par exemple un retard, une difficulté ou un aléa dans la production). L'espace participe de la communication non verbale. Par les relations qu'il permet ou non, il vient soutenir ou contraindre le travail. Les figures 1 et 2 viennent illustrer ce propos ${ }^{2}$.

2. L'espace agi comme dimension émergente de l'activité au travers des relations, coordinations, coopérations et toutes les formes de communication verbale ou non qui se créent lors de la réalisation de l'activité (Figure 3). Il remodèle la disposition des lieux en débordant les postes et les locaux prévus par les concepteurs ou les décideurs, conduisant ainsi à reconfigurer la structure spatiale proposée par l'organisation selon une logique organisée par l'activité de travail. L'ensemble forme un périmètre mettant en lien différents espaces (de travail, de circulation, de rangement, de pause, etc.) concourant, d'une manière directe ou indirecte, à la réalisation de l'activité de travail. Il est constitué d'espaces supports pour l'activité individuelle et collective. Ce sont des espaces ressources pour les échanges et les régulations entre collègues ou avec la hiérarchie. La délimitation de cet ensemble spatial est circonscrite par l'activité qui le façonne. Ce système, en intégrant tous les espaces et les relations (visuelles, sonores et déplacements physiques), participe de la production. Il constitue une unité reliant activité de travail et espace. Il n'est pas défini par la tâche, mais par la dynamique de l'activité dans l'expérience sensible et incarnée du travail. La figure 3 schématise ce système qui émerge dans la manière dont l'activité de travail prend place dans un lieu. Lors du déroulé du travail réel, les espaces sont reliés dans des continuités relationnelles dans une logique propre à l'activité. Cet ensemble déborde et reconfigure les découpages opérés par la prescription ou la conception. Il fonde sa cohérence du point de vue du travail. Il est une déclinaison spatiale de la situation de travail. L'espace de l'activité spatialise la situation de travail. Ce système, relatif à l'activité de travail, est schématisé dans la figure 3, par un aplat de couleur vert clair.

Figure 1 : L'espace donné : l'espace du poste de travail et du local.

Figure 1: Given space: the space of the workstation or the workspace

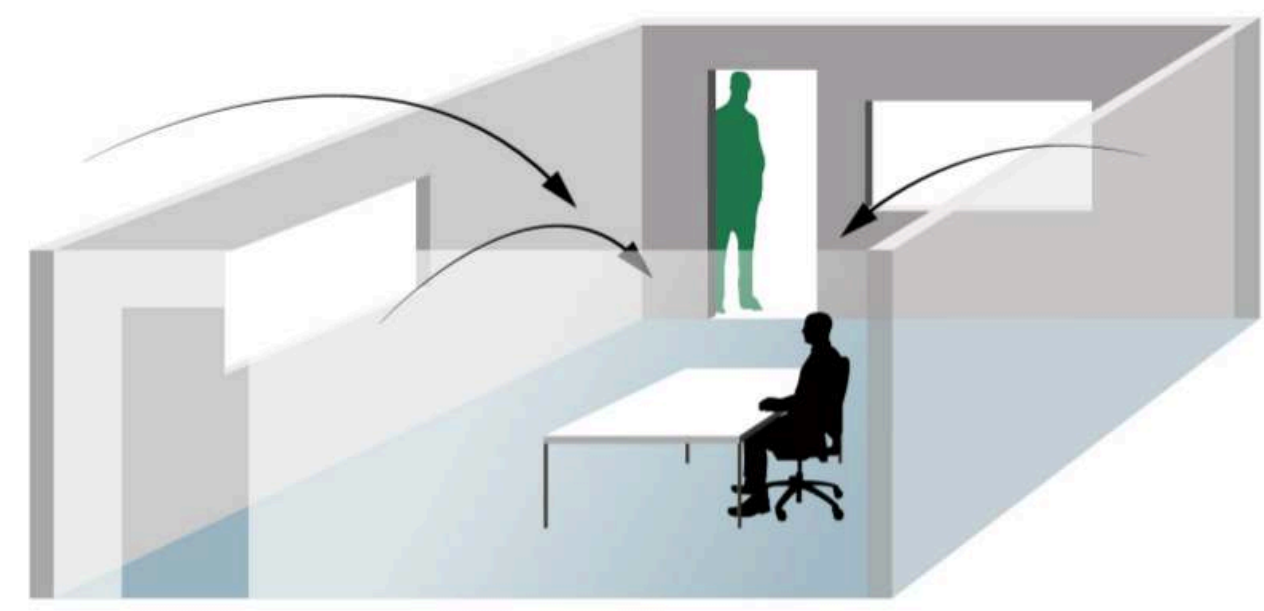


Figure 2 : L'espace modifié et ajusté par l'activité.

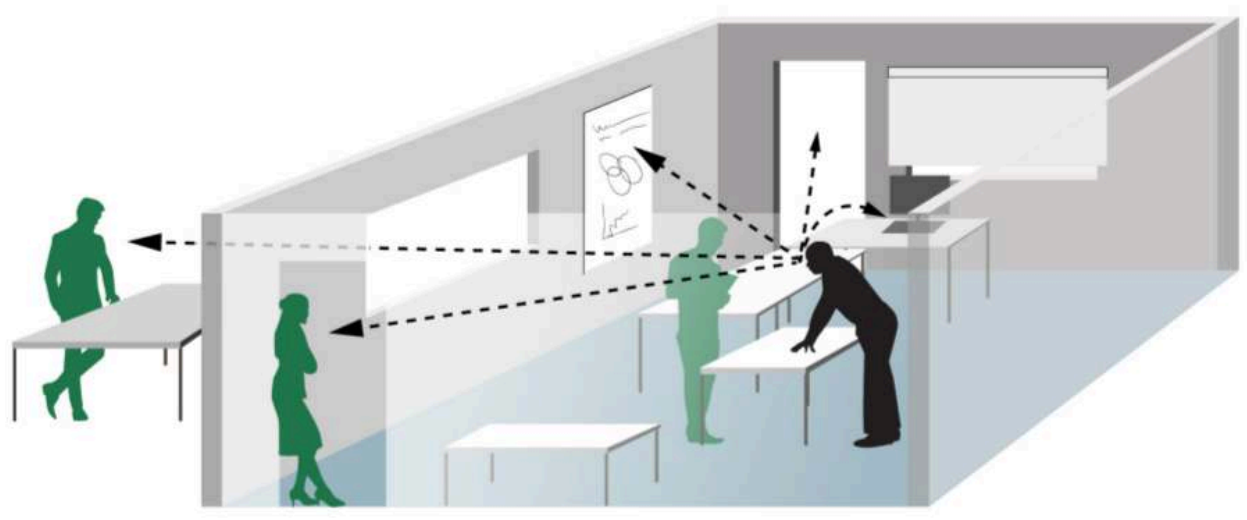

Figure3 : Illustrations de l'espace émergeant. Figure 3: Illustrations of emerging space

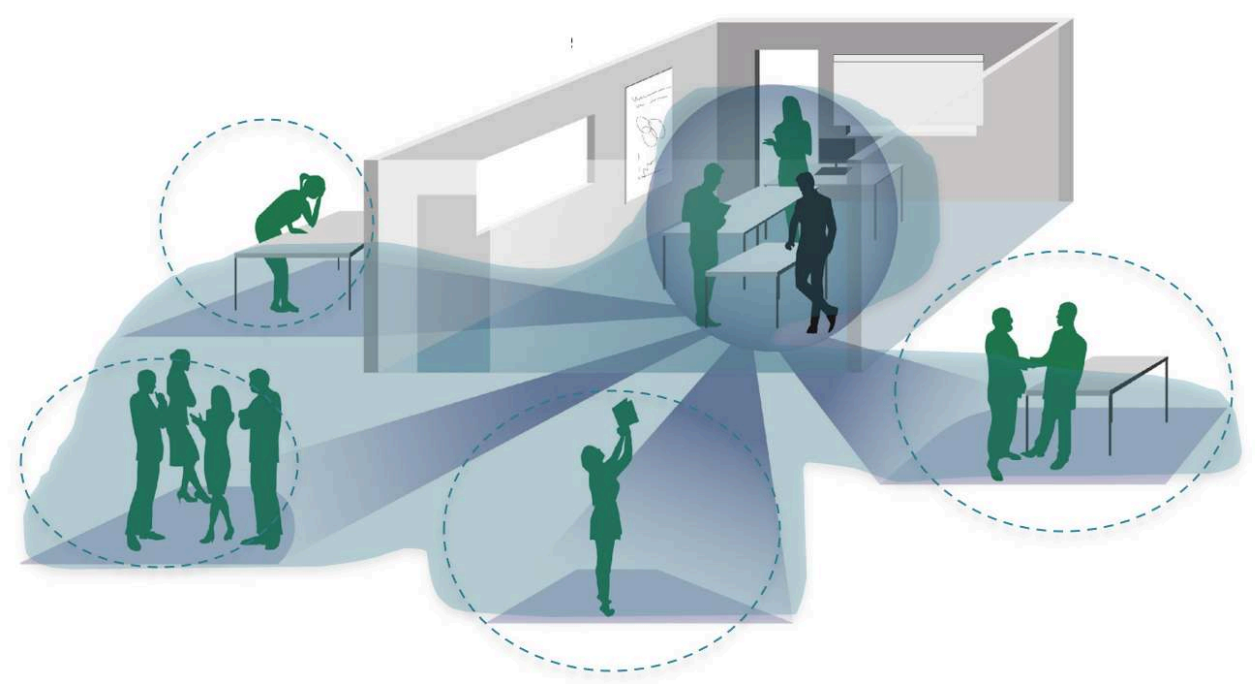

Pour conclure ici, le périmètre de l'espace de l'activité invite à saisir l'espace agi par l'activité et questionner le découpage classique (postes et locaux) opéré dans les organisations du travail. D'une certaine manière, l'espace de l'activité, rapportée aux lieux de travail, rejoint la notion de milieu développée par Ricœur (1969), Canguilhem (1943), mais aussi Berque (2009) pour qui l'homme et son milieu se génèrent et se modèlent l'un et l'autre, et cela de façon réciproque. Cet espace ne se comprend que par une analyse de l'activité imbriquée à une analyse spatiale de l'activité de travail.

\section{La question de l'introduction massive du numérique dans les espaces dédiés au travail}

La technique a de tout temps fait partie intégrante de l'évolution de l'homme comme moyen d'agir sur la nature (Simondon, 1958). Elle est considérée par certains auteurs comme une forme d'extension du corps de l'homme (Hall, 1971).

Si la naissance des technologies de l'information et de l'intelligence artificielle (IA) est située dans les années 50 et que les études qui traitent du lien entre IA et travail ne sont 
pas nouvelles (Zouinar, 2020), la question de la relation entre numérique et espaces de travail est peu explorée.

\section{1. Évolutions technologiques et espaces d'entreprise}

Les espaces sont fortement investis par la technologie numérique depuis la fin des années 90 modifiant profondément son lien au travail. Les technologies contribuent à relier différemment les personnes au travail. La proximité spatiale n'est plus une condition pour travailler ensemble. L'unité du lieu historiquement requise pour une action collective n'est plus une condition. La collaboration, le contrôle et les échanges pouvant se faire sans réunir, dans un même lieu, les équipes qui peuvent être en lien, tout en étant spatialement dispersées. La technologie permet, d'une certaine manière, un affranchissement de l'espace et du temps avec l'idée qu'il est possible de travailler partout et à tout moment. Les organisations de travail s'équipent et investissent massivement dans toutes sortes de technologies contribuant au développement de la production, des modalités de suivi et de contrôle dans une logique de recherche de performance économique.

Dans ce contexte, il est possible d'interroger la place du numérique dans les configurations spatiales des organisations du travail. Est-ce une technique comme une autre? Quelle est sa particularité ?

Dans les organisations de travail, les technologies numériques viennent prolonger l'idée d'un espace a priori qu'il faut équiper, transformer ou supprimer selon les choix en matière de technologie de communication. L'offre spatiale dans les entreprises se décline en un espace conçu comme un contenant (ou un support) qu'il faut compléter par un ensemble d'outils de travail dont le numérique occupe une place centrale.

Pourtant, implanter des moyens numériques modifie le rapport à l'espace. Se faisant, il contribue à transformer l'espace dans les entreprises. L'espace est en effet fortement modifié au travers des relations en présence ou à distance entre les personnes en situation de travail. Les modalités des relations et des coordinations sont modifiées par l'éclatement de l'unité spatiale et temporelle historique de l'entreprise. Le recours aux technologies conduit à franchir le périmètre spatial et temporel de l'entreprise.

De plus, une double évolution peut s'observer. D'un côté, les clients sont introduits directement dans l'organisation (Gadrey \& Zarifian, 2003) qui doit s'ajuster. Autrefois à l'extérieur de l'entreprise, le client est invité à intervenir directement sur les formes de production en formulant des demandes et en contribuant à agir directement sur les processus de production au travers des systèmes techniques qui l'amènent à renseigner des données relatives à sa demande ou ses attentes. De l'autre, le numérique incite les organisations à développer de nouveaux services en dehors de son périmètre spatial et temporel habituel. Dans le temps, les espaces propres à l'entreprise évoluent. La frontière séparant historiquement clairement un dedans du dehors est chahutée. Cette évolution touche tous les espaces de production. 


\section{2. Évolution de l'immobilier d'entreprise dans le secteur tertiaire : introduction du modèle spatial du flex office} favoriser les échanges par l'arrivée massive des technologies dans les organisations du travail. Le numérique viendrait se substituer au besoin d'un espace dédié pour réaliser le travail. En étant partagé, l'espace (surfaces et postes) peut être économisé. Le flex office s'installe comme modèle dans le tertiaire. 


\section{Présentation de l'étude de cas : un service aménagé en flex office}

51 L'étude de cas a été réalisée dans le secteur tertiaire, dans les services du siège d'une grande entreprise de télécommunications française ${ }^{3}$.

Suite au constat d'une occupation temporelle partielle des postes de travail par le chef de service, un réaménagement sur le modèle du en flex office est proposé en 2016 par un cabinet de conseil spécialisé dans l'aménagement tertiaire. Ce choix est essentiellement motivé par la question du temps d'occupation des postes de travail par les collaborateurs, souvent en réunion, en déplacement ou en télétravail. Supprimer le poste de travail au bénéfice d'espaces partagés constitue ainsi pour l'organisation une réponse au nombre de postes de travail jugés trop souvent comme inoccupés.

Par ailleurs, la volonté d'offrir un aménagement visant à rompre avec l'espace bureau classique est avancé pour satisfaire deux critères selon le chef de service. Le premier répond à un enjeu d'affichage. Face à la concurrence, il s'agit de montrer que l'entreprise sait innover en affichant des espaces atypiques et innovants du point de vue de leurs agencements et des mobiliers. Le second critère vise à satisfaire les attentes des jeunes talents que l'entreprise souhaite attirer. Il s'agit de plaire à la génération née avec l'installation durable du numérique dans la société.

Le choix du modèle du flex office est précédé par l'équipement de tous les salariés de matériels informatiques et téléphoniques portables. Ils ont tous été dotés de tablettes, téléphones et ordinateurs portables avant la mise en place du nouvel agencement spatial. Les pratiques individuelles et collectives de travailler en dehors des espaces de l'entreprise ont été installées avant l'installation des équipes dans le nouvel espace.

\subsection{Contexte de la demande}

La demande a porté sur la réalisation d'un retour d'expérience suite à la mise en place d'un modèle spatial du "flex office " pour spatial et temporel de laquelle la référence n'est plus le poste de travail qui disparaît au profit d'une collection de zones et espaces variés. Cette analyse a été réalisée en 2017, 18 mois après l'initiation du projet ayant impliqué le personnel et 6 mois après l'installation physique des équipes dans le nouvel aménagement proposé par le cabinet spécialisé dans les aménagements tertiaires.

La demande est à l'initiative du chef de service qui souhaite réaliser un état des lieux à l'issue du projet du nouvel aménagement avec pour enjeu de s'assurer de la pertinence du modèle spatial adopté, mais aussi pour instruire les éventuels effets de ce nouveau modèle spatial sur le travail. La décision de réaliser une analyse post installation est motivée par la volonté du chef de service de recourir à un point de vue extérieur, sans lien avec l'organisation hiérarchique, ni avec les acteurs ayant contribué à la conception de l'aménagement. L'équipe concernée par cette étude est constituée d'une trentaine de personnes dont une grande majorité est constituée de cadres, chargés de projets dans l'immobilier. Ce sont, pour une grande part, des hommes et près des $3 / 4$ ont plus de 46 ans. 


\subsection{Méthodes} catégories de caractéristiques relatives à la question des espaces sur le modèle du flex office:

- Un espace de la tâche centré sur la technique et une approche temporelle de l'occupation des locaux.

- Un espace de la tâche imprégné des codes symboliques de la sphère privée brouillant les frontières entre espaces.

- Des espaces investis de plus en plus en dehors de l'entreprise contribuant à modifier les limites temporelles et spatiales du travail.

- Un recours renforcé du numérique par travail dans un espace en flex office. les concepteurs participe de la tâche. La troisième met l'accent sur la porosité spatiale et temporelle du travail qui s'opère de plus en plus en dehors des lieux de l'entreprise. La dernière caractéristique concerne les formes de coordinations nécessaires à la réalisation du travail dans le modèle du flex office, engendrant ainsi une double dépendance en amont et en aval de la conception: le numérique est à la fois une condition et une résultante pour le travail dans une espace en flex office. 


\subsection{Un espace conçu à partir de la technique et l'occupation temporelle des espaces}

Cela a été évoqué, dans le modèle du flex office, les entreprises suppriment le poste de travail, jusque-là individuel et attribué, pour proposer des positions de travail non dédiées dans un modèle spatial, dit «flex" pour signifier une flexibilité. Ce modèle spatial ne modifie en effet pas structurellement la figure historique de l'espace ouvert (l'open space), mais il le prolonge en introduisant une rupture dans le rapport au poste de travail. D'une organisation spatiale historiquement composée d'une somme de postes de travail dédiés, les entreprises font aujourd'hui le choix d'une collection d'espaces différents et partagés dans une logique d'usage ponctuel.

Du temps long d'occupation d'un poste de travail, les organisations du travail optent pour le temps court d'usage d'espaces différents. La focale est mise sur les temps de présence au sein de l'entreprise avec le choix de catégoriser, non pas la nature du travail réalisé, mais le profil des personnels selon des archétypes d'occupation temporelle.

Ainsi, dans le cas de la grande entreprise française de télécommunications, sont identifiés par l'organisation du travail différents profils utilisateurs du poste de travail :

- Un profil sédentaire pour lequel le temps est estimé à $60 \%$ du temps passé en tâche d'analyse et de concentration, $25 \%$ du temps en réunion et $15 \%$ du temps pour les tâches de débriefing informel, question / réponse (travail dit collaboratif);

- Un profil semi-nomade pour lequel le temps est estimé à $30 \%$ du temps passé pour les tâches d'analyse et de concentration, 35 à $45 \%$ du temps est passé en réunion, $20 \%$ du temps pour les tâches dites médias (téléphone, mails, échange d'information) et $10 \%$ du temps pour les tâches confidentielles ;

- Un profil nomade pour lequel le temps est estimé à $20 \%$ du temps pour les tâches d'analyse et de concentration, $60 \%$ du temps en réunion, $10 \%$ du temps en activité dite médias (téléphone, mails, échange d'information) et 10 \% du temps pour les tâches confidentielles.

5 Ces profils sont constatés par le cabinet spécialisé en aménagement intérieur, à l'aide de questionnaires dirigés et de techniques de relevé et de comptage des temps de présences des personnes dans les locaux.

6 Lors des études de conception du nouvel aménagement en flex office, tous les collaborateurs ont été invités à se prononcer sur la nature de leurs tâches à partir d'un nuancier de couleur (Figure 4). Il distingue les tâches à réaliser hors bureau, dans un environnement feutré et calme et celles qui reposent sur des interactions. Une couleur est attribuée pour chaque typologie: le bleu illustre les tâches de concentration, le rouge, les tâches d'interaction et le gris, les tâches effectuées en dehors du bureau.

Dans le cas de cette entreprise, seul 1 sur 5 des collaborateurs a été identifié par le cabinet au travers du recours à ce nuancier, comme réalisant un travail sédentaire. Il s'agit des fonctions supports, principalement constituées des assistants et du directeur de l'administration et de la performance.

Toujours selon le nuancier, les collaborateurs sont majoritairement identifiés comme «nomades» ou «semi-nomades». Les tâches qui leur sont assignées sont de nature majoritairement relationnelle. Elles sont réalisées à l'extérieur ou dans l'entreprise, notamment dans les salles de réunions. 
Figure 4 : Illustration du nuancier. Figure 4: Illustration of the color chart

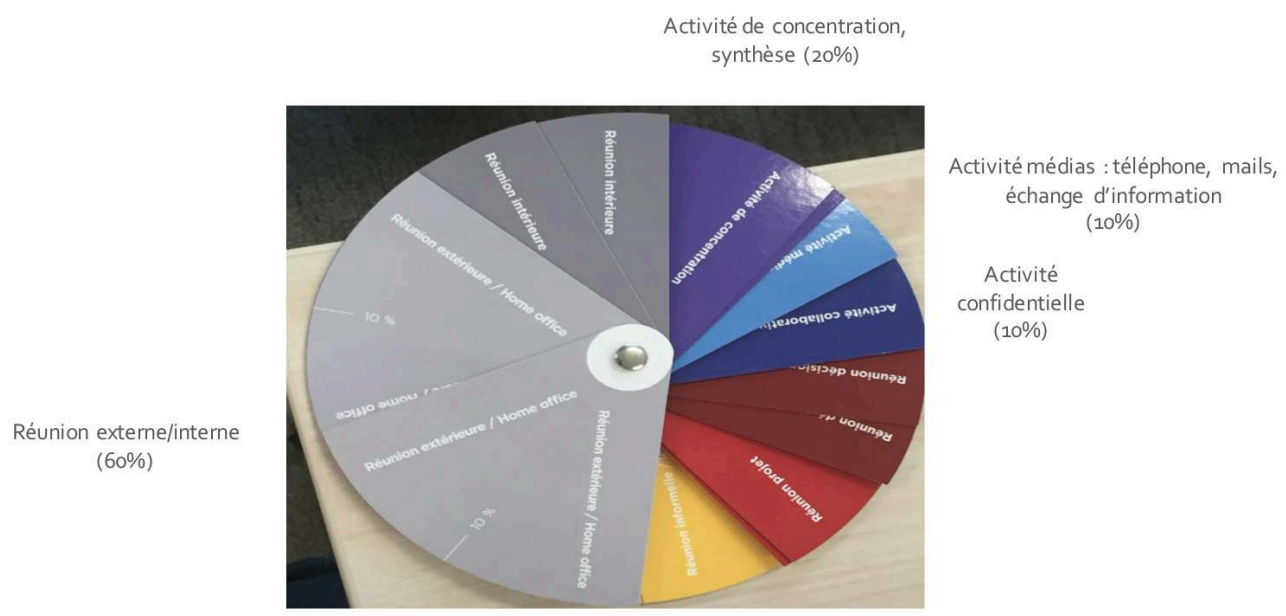

Par exemple, la figure 4 illustre un profil correspondant à $60 \%$ du temps consacré aux tâches réalisées à l'extérieur, 10 \% à des tâches de concentration et de synthèse, 10 \% à des tâches en lien avec des échanges (téléphone et email majoritairement) et 10 \% à des tâches consacrées à la gestion d'informations confidentielles qui requièrent un espace fermé. Le nuancier pointe ici un profil «nomade».

Selon la proportion des profils identifiés via ce nuancier, le choix du nombre et de la typologie des espaces est réalisé. Au total, 11 types d'espaces différents ${ }^{5}$ ont été proposés par le cabinet spécialisé en aménagement pour un service composé d'une trentaine de personnes. Les personnels ont été associés dans le choix des mobiliers et espaces sur la base de catalogues de mobilier et à l'issue de deux visites d'entreprise ayant adopté le modèle du flex office.

Parmi les propositions, il est possible de relever: des grands espaces ouverts comprenant des plans de travail partagés (de type bench) en posture assis-debout, des espaces ouverts avec des plans de travail partagés en posture assise, des zones avec des fauteuils en forme d'alcôves pour le travail individuel ou un travail à deux, des espaces dédiés à la concentration prévus fermés et traités acoustiquement, des boxes dédiés pour les appels téléphoniques disposés dans les espaces partagés, un coin salon, une cuisine, etc. Chacun de ces espaces se distingue par son emplacement, son mobilier et ses équipements spécifiques ${ }^{6}$ répondant chacun à un mode d'utilisation dans une logique temporelle.

La généralisation du numérique ayant précédé le projet d'aménagement, les moyens matériels et organisationnels pour l'utilisation des différents outils numériques dans la réalisation du travail sont envisagés dans tous les espaces. L'ensemble de la documentation est dématérialisé et accessible en ligne via un système de Gestion Électronique des Documents (GED) et tous les locaux disposent de moyens de connexions filaires (électricité et réseau Ethernet) et non filaires (Wifi). Les espaces collectifs « projets » sont équipés de larges écrans disposés sur un mur pour le partage des supports et certaines zones, dites d'idéation, de grands tableaux blancs interactifs. Les collaborateurs peuvent librement disposer des locaux et s'installer où ils le souhaitent selon ce qu'ils ont à réaliser. Équipés d'un téléphone, d'un ordinateur et d'une tablette portables, ils sont ainsi invités à se déplacer plusieurs fois dans la même journée pour utiliser les différents espaces selon la nature de la tâche à réaliser. Il leur 
est demandé de ne pas laisser des effets personnels ou de travail dans les différentes zones de travail lorsqu'ils ne sont plus présents dans les lieux. Des casiers ont été prévus pour le rangement des documents ou matériels personnels. Avec l'installation en flex office, le rangement est à réaliser pendant la journée, s'ils se déplacent d'une zone à l'autre, et en fin de journée afin de laisser la place libre pour tout autre collègue. Les postes de travail doivent en effet, être accessibles à tous, tous les jours. Chacun doit pouvoir occuper une zone ou une autre selon ce qu'il a à réaliser (travail de concentration, d'échange et d'interaction ou de coordination).

74 L'intérêt d'une catégorisation temporelle des profils des personnels est qu'il permet ici de gérer au mieux les besoins en surface. Le nombre de positions de travail prévu par l'agencement est calculé sur la base d'un ratio. Ce ratio est arrêté selon le taux de présence physique dans les locaux à partir d'un comptage du nombre de personnes qui occupent les espaces. Ce ratio varie généralement d'une entreprise à l'autre et vise à optimiser les surfaces dédiées au travail au niveau individuel et collectif avec l'idée de remettre en commun un maximum de zones et espaces. Considérant que l'ensemble du personnel n'est jamais dans le lieu en même temps, ce taux est généralement inférieur à un. En offrant une diversité d'espaces aménagés et équipés différemment pour des usages différenciés, l'espace, conçu sur le modèle du flex office, s'inscrit dans une économie de la fonctionnalité privilégiant une vision temporelle de l'occupation des espaces dans l'horizon court de la journée de travail.

Ce modèle s'accompagne par une tendance immobilière qui ne se base plus sur la propriété, mais sur la location des surfaces. Les entreprises investissent aujourd'hui, de moins en moins dans un bâti pensé pour elles, mais font le choix de louer des surfaces pensées en "flex ». Il s'agit d'une forme de rationalisation des espaces à partir d'un modèle spatial pouvant convenir au plus grand nombre. Cette tendance qui consiste à choisir sur catalogue une typologie d'espaces différents permet de faire l'économie d'une adaptation ad hoc des locaux à la spécificité non seulement de l'entreprise, mais aussi de la nature de l'activité des équipes. Le travail est découpé en grandes catégories prédéfinies de zones (de concentration, d'échange, de travail en mode projet et même de créativité) à partir d'une lecture des temps alloués aux différentes tâches.

\subsection{Un espace de la tâche imprégné des codes symboliques de la sphère privée}

La tendance de proposer une collection variée d'espaces différenciés s'accompagne par le recours à des aménagements et une décoration qui s'inspirent largement des codes de la sphère privée. Aux plans de travail de type bench, se rajoutent des zones aménagées avec des plans de travail étroits et hauts, des comptoirs équipés de sièges de type bar, de nombreux espaces salons, des espaces de détente et une cuisine.

77 Le panel large des typologies d'espaces offerts propose tout aussi bien des plans de travail collectif, des positions pour le travail individuel disposées dans des fauteuils équipés des connexions électriques et de réseaux, des fauteuils pour échanger ou accueillir, des transats pour travailler individuellement ou collectivement.

Les choix de couleurs et des matériaux s'inspirent de codes symboliques de la sphère privée. Des fauteuils, parfois des poufs, sont disposés sur des tapis. La cuisine est équipée d'un grand plan de travail avec des possibilités de connexion aux réseaux 
(prises électriques, wifi, etc.). Un hamac est disposé dans un coin. Un babyfoot est proposé dans la salle de détente.

Ces choix visent à créer une ambiance qui se veut conviviale. Les concepteurs recherchent un code spatial permettant de se sentir explicitement «comme à la maison", une terminologie adoptée largement dans les documents de présentation pour expliquer les choix du projet en matière de mobilier et de décoration. Ces vocables ont été repris par l'encadrement qui les a relayés lors des entretiens réalisés dans le cadre de cette démarche. Adopter un mobilier pour faire « comme à la maison » viserait à rompre avec le modèle du bureau classique dans le tertiaire. L'objectif recherché consiste à inviter les collaborateurs à un usage moins conventionnel et plus convivial des espaces proposés par l'entreprise.

Les observations réalisées dans cette grande entreprise de télécommunications ont montré des personnels habillés en costumes s'installer dans des sofas ou des fauteuils disposés face à des tables basses. La posture peut être détendue dans un fauteuil, mais à la vue d'un responsable, il est aisé de les observer se redresser rapidement pour éviter un air avachi ou engoncé dans un sofa. En effet, si le mobilier invite à des postures de détente, il leur est difficile de se comporter comme dans la sphère privée. Les collaborateurs gardent un œil sur les personnes qui entrent et sortent de l'espace, évitent de retirer les chaussures ou mettre leurs jambes sur un pouf. Même installées confortablement dans un sofa, il leur est difficile de se soustraire du poids de la hiérarchie. Des salariés évitent même certains espaces ou mobiliers pouvant les mettre dans une posture inappropriée face à un responsable, d'autres demeurent en alerte pour se redresser à tout moment en cas de passage d'un hiérarchique dans les espaces. Certains choisissent de se placer à des postes qui leur permettent un visuel sur qui entre ou sort du local de façon à anticiper sur la manière de se tenir selon les personnes qui entrent dans le service. Ils se placent ainsi prioritairement dans les zones et sur les positions de travail qui leur donne accès à une capacité de surveiller les entrées et sorties du service et ainsi se préparer à engager ou non une relation, une attitude face à la personne qui entre dans le service. Ces emplacements, identifiés par des collaborateurs, notamment les plus âgés et expérimentés, permettent une forme de vigie sur ce qui se passe dans le service. Ils sont souvent les plus rapidement occupés tôt le matin.

81 Ces stratégies d'occupation des différentes zones du flex office montrent que la convivialité recherchée par les choix d'agencements n'efface pas la question de la subordination du travail dans l'organisation hiérarchique qui caractérise cette grande entreprise de télécommunications. Elle met en tension l'intention de convivialité recherchée par les concepteurs de l'aménagement et le modèle organisationnel qui demeure fortement hiérarchique dans l'organisation travail. De ce point de vue, le comportement est différent selon l'expérience et l'ancienneté des collaborateurs. Ce sont en effet, les personnels les plus âgés et expérimentés qui soulignent le besoin de prendre quelques précautions dans la manière de se tenir et se placer dans le service. Les plus jeunes, moins attentifs selon les plus expérimentés, sont plus exposés aux difficultés relationnelles qui peuvent survenir en cas de conflit, notamment face aux normes implicites de la hiérarchie qui évalue malgré tout, tout aussi bien les résultats du travail que les comportements spatiaux dans le flex office. Les codes symboliques spatiaux empruntés à la sphère privée n'effacent pas la place de chacun dans l'organisation du travail. Le "comme à la maison", plébiscité par la conception, 
brouille au final les codes comportementaux à adopter dans les espaces, moins intelligibles par les jeunes recrues, sans expérience des relations de travail, notamment dans un rapport de subordination. Il les expose.

\subsection{Des espaces investis de plus en plus en dehors de l'entreprise brouillant les frontières temporelles et spatiales du travail}

Le recours massif aux nouvelles technologies numériques, combiné à la mise en place d'un nouveau modèle d'espace basé sur la non-attribution d'un poste de travail pour chaque salarié, conduit à l'élaboration de nouvelles formes de recours aux espaces au sein et en dehors de l'entreprise. Après six mois d'expérience du flex dans le cas de l'entreprise de télécommunications, les résultats des entretiens individuels et collectifs montrent le développement d'un sentiment d'une présence générale dans les locaux qui s'amenuise dans le temps au sein de l'équipe. Ce constat est réalisé en particulier par les collaborateurs reconnus par l'organisation comme des «nomades » ou « seminomades", c'est-à-dire les $4 / 5 \mathrm{du}$ personnel dans le cas de cette entreprise de télécommunication. En pratiquant eux-mêmes de moins en moins les locaux de l'entreprise, les collaborateurs constatent que les collègues, qu'ils espèrent croiser dans le service, sont eux aussi de moins en moins présents dans les espaces de leur service d'appartenance. Selon leur expression, le service ne serait plus «le QG, le quartier général » de l'équipe. En étant très souvent en déplacement, pratiquant le télétravail et accédant à toute la documentation en ligne, la raison évoquée est qu'il n'a plus de raison à regagner le bureau. " Avant, je revenais toujours au bureau, même tard le soir pour déposer des dossiers, en récupérer d'autres, mais aussi pour croiser des collègues. Aujourd'hui, j'ai accès à tout depuis mon téléphone ou portable. Au bureau, je n'ai plus d'endroit pour laisser des affaires et je ne suis plus certain d'y croiser des collègues ", explique un salarié. Déposer ou récupérer un dossier au bureau constitue en effet une opportunité pour croiser des collègues.

Par ailleurs, ne disposant plus que d'un casier au bureau, les collaborateurs pratiquant les tiers lieux, dont le domicile avec le télétravail, investissent leur habitation pour stocker les affaires. Ils développent également des pratiques d'occupation des salons voyageurs des gares et des aéroports ou encore les cafés et autres espaces atypiques offerts par l'espace urbain.

L'unité spatiale relative au travail est reconfigurée individuellement en mêlant les périmètres travail, hors travail et ville brouillant les frontières dedans/dehors de l'entreprise. L'espace proposé par l'entreprise ne constitue plus un ancrage à l'activité, il se prolonge d'une constellation d'espaces extérieurs à l'entreprise. La production est réalisée dans des espaces privés, professionnels ou publics.

\subsection{Un recours renforcé du numérique par le travail dans l'espace en flex office}

85 La manière de s'installer dans le nouvel aménagement en flex office repose sur la capacité de chacun à anticiper les tâches à réaliser et leur déroulé précis dans le temps. Organiser une réunion, formelle, dans une salle réservée, ou informelle, dans l'un des différents espaces disponibles dans le service, suppose, dans les deux cas, de spécifier le sujet à traiter, le déroulé temporel des tâches à prévoir, les conditions matérielles pour 
leur réalisation et le nombre précis de participants à considérer. Décider ainsi de se retrouver, dans un coin salon, dans un espace projet ou dans une zone de la cuisine, consiste à projeter le déroulé temporel de la rencontre et les moyens matériels pour sa mise en œuvre. Le travail en commun, dans un espace précis, repose ainsi sur une préparation de ses conditions organisationnelles, techniques et spatiales. Selon le travail à réaliser, le choix de l'espace est différent ${ }^{7}$. Il s'agit ici d'une nouvelle tâche. La coordination dans la manière de recourir à la ressource spatiale au sein de l'entreprise pour composer avec les moyens disponibles mis à la disposition de tous est ainsi une nouvelle exigence du travail. Elle s'appuie sur une régulation entre les salariés pour l'utilisation des locaux. Le risque que certaines espaces ou zones de travail soient plus sollicités que d'autres n'est cependant pas écarté. Certains collaborateurs arrivent très tôt le matin afin d'avoir le choix des places, mais aussi pour occuper certaines positions de travail plus appropriées pour travailler avec des collègues qui arrivent plus tard dans la journée. La gestion de cet équilibre est à la charge des équipes qui doivent anticiper les moyens pour décider d'une réunion dans un lieu précis ou au travers des outils de communication. La rencontre pour le travail en commun repose ainsi sur une préparation de ses conditions organisationnelles, techniques et spatiales. Ils ont recours aux outils numériques pour planifier, coordonner les échanges et leurs modalités pratiques qu'elles soient matérielles (dans un espace) ou immatérielles (via les outils de communication). Ils y ont également recours pendant le travail en commun par exemple, pour accéder à de la ressource documentaire en ligne ou pour entrer en lien avec d'autres collaborateurs via un échange vocal ou visuel. Les deux moyens, matériels (espace, équipements et mobiliers) et immatériels (via les applications), s'imbriquent.

Dans un flex office, le numérique vient ainsi soutenir comme il vient remplacer une relation dans un espace physique.

Enfin, une autre observation est fortement appuyée par les collaborateurs lors des entretiens. Ils signalent tous rechercher les collègues dans les espaces de l'entreprise depuis la mise en place du flex office. Une majorité d'entre eux s'accorde à noter que le flex office a conduit à une rupture dans la manière dont chacun peut se représenter la localisation des uns et des autres dans l'espace dédié au travail au sein du service. "Avant, je savais où les collègues étaient installés. Avec le flex, je les cherche beaucoup. » Le recours au téléphone portable et à la messagerie est généralisé. Même en étant certains que le collègue est dans les lieux, les salariés précèdent la rencontre par l'envoi d'un message via un texto ou un email. Ils s'assurent ainsi que le rendezvous a bien lieu, mais surtout, ils s'informent de la localisation de chacun dans l'espace du flex office.

Rechercher les personnes, installées quelque part dans une des zones du flex office, constitue un effet de l'organisation spatiale. Il s'agit aussi d'un facteur qui conduit à davantage utiliser les outils de communication offerts par le numérique.

D'une certaine manière, le numérique, une condition ayant permis un passage aisé en mode flex office de l'organisation des espaces, se retrouve renforcé dans sa généralisation et son utilisation au travers des personnels qui l'utilisent davantage, notamment pour gérer les effets du flex office. D'une condition initiale (permettre une mobilité des salariés dans toutes les zones en supprimant le poste fixe), le numérique acquière le statut de ressource pour prendre en charge un effet auquel il aura lui-même contribué. 


\title{
7. Discussion
}

\author{
tertiaire interroge deux registres :
}

\subsection{Les effets du développement imbriqué du numérique et de l'espace}

91 Cette évolution conduit sur le plan des résultats, à trois effets pouvant être discutés.

\section{Une décorrélation entre la production et le lieu}

Historiquement, dans toute organisation du travail, la mise à disposition des moyens matériels vise à soutenir le travail de production. Machines, équipements, outils, postes, locaux, etc., participent de la production. Les espaces sont envisagés comme faisant partie du système productif. En rassemblant les moyens et les personnels pour que la production prenne place, l'espace dédié à l'activité de travail fait unité de lieu. Non seulement les postes de travail sont affectés aux employés, mais les matériels sont localisés. L'organisation du travail a pour charge d'organiser et coordonner l'affectation et l'utilisation des moyens tout en attribuant une place à chacun. Il s'agit ici de l'espace de la tâche, il est composé des moyens matériels et spatiaux.

Avec l'arrivée de l'ordinateur et du téléphone portable, associée au développement des réseaux non filaires et la dématérialisation des documents, la question de la place physique des salariés dans une organisation spatiale est remise en cause. Attribuer un poste fixe n'est plus une condition pour la réalisation de la production, ni pour contrôler les résultats du travail des employés. Les entreprises font aujourd'hui le choix d'équiper les personnels de toutes sortes de matériels et outils numériques (ordinateurs, tablettes et téléphone portable, applications ou systèmes pour les échanges et réunions à distance, dématérialisation et gestion électronique des documents, etc.) permettant le travail en dehors de l'entreprise. Le travail chez le client, dans un salon d'attente d'une gare ou d'un aéroport, dans un café, un bar ou à domicile est non seulement possible, il est encouragé.

Le développement du numérique a contribué à décorréler la production du lieu. Ce faisant, il interroge la place de l'espace de l'entreprise, notamment son rôle dans une organisation où la production peut se réaliser partout et à tout moment. Cette situation s'observe en particulier dans le tertiaire. Un secteur où la production est pour une grande partie aujourd'hui dématérialisée et peu visible dans l'espace.

Les locaux dans les entreprises sont parfois même occupés par les techniques et les personnes de façon indifférenciée, mais l'espace de la tâche évolue cependant avec une importance forte accordée à la technologie comme socle des échanges et des communications dans le tertiaire. C'est la technique qui relie, rassemble et non plus les espaces dont la fonction était jusque-là de rassembler et unir. Les flux d'information circulent, les données sont accessibles à distance et les modes de coordination intègrent tant les systèmes techniques que les personnes, en temps réel ou différé et dans un espace séparé ou non. Le travail se réalise tant au domicile que dans les trains, les cafés que chez les clients ou dans l'entreprise. 


\section{Une standardisation des lieux dédiés au travail dans et en dehors de l'entreprise}

papier circulant d'un poste à l'autre ou d'un espace à l'autre était du ressort de l'encadrement, les locaux tertiaires ont toujours souffert d'une forme de traduction spatiale visuellement homogène, voire pauvre. En effet, les espaces bureaux se ressemblent: ils sont composés de tables, chaises, ordinateurs, imprimantes et rangements pour les dossiers ou la documentation en papier.

Cette homogénéité des moyens matériels spatiaux conforte l'invisibilisation de l'activité réelle des personnels. Elle masque pourtant une diversité de situations de travail. En effet, produire au sein d'un service de comptabilité n'est pas réaliser l'activité dans une entité commerciale du point de vue des personnes qui réalisent le travail. Pourtant, cette vision homogène des lieux est portée pour les acteurs en charge de la conception et la mise à disposition des moyens techniques et spatiaux. Cette représentation contribue probablement à accorder une place centrale au numérique dans la conception des espaces. Les choix d'espace ne résultent ainsi plus des critères de production, mais des possibilités offertes par la technologie numérique. D'une somme de postes de travail dédiés à des salariés ou à la réalisation de tâches spécifiques, l'organisation spatiale propose une collection d'espaces distincts non attribués dans le cadre du flex office. Le catalogue mobilier et le cahier des tendances (couleurs, décoration, etc.) deviennent une référence ${ }^{8}$ et la question de l'organisation du travail de production passe au second plan.

aurs, la tendance à concevoir les espaces des entreprises en faisant référence à des codes symboliques de la sphère privée, conduit non seulement à une forme de perte de repères, mais aussi à homogénéiser les choix des mobiliers, des ambiances et les traitements décoratifs des locaux. Les espaces, dans et hors de l'entreprise, mettent en avant des salons équipés d'alcôves ou de fauteuils connectés, une cuisine ou des espaces dotés de benchs. Le rapport à l'espace, pour réaliser la production, se standardise d'une certaine manière. L'expérience spatiale en entreprise ne se distingue plus de celle d'un tiers-lieu ou d'un salon voyageurs d'une gare ou d'un aéroport. Les formes d'agencement des espaces et les mobiliers conduisent à une normalisation des lieux qui se ressemblent.

\section{Une reconfiguration interrogeant la question de la limite spatiale et temporelle du travail}

En effet, le numérique conduit à modifier structurellement les relations entre le corps, l'espace et la nature de l'activité de travail. Il ne supprime pas la relation au corps, mais il la transforme au-delà de l'espace physique pour englober des espaces d'échange virtuels qui modifient l'activité. Le travail se réalise individuellement et collectivement tant au domicile que dans les gares, aéroports, bars que chez les clients ou dans l'entreprise. Pour l'entreprise, la mise en relation entre des collègues se fait tant dans un espace physique que via les outils numériques. Les espaces dans lesquels se réalise le travail débordent le périmètre de l'entreprise. Cet éclatement de l'unité spatiale questionne la limite. En dématérialisant les relations et la production, les frontières entre la vie privée et professionnelle s'estompent. Où commence et s'arrête le travail de production dans ce contexte? 
100 Pour conclure sur la question des effets, il est possible d'interroger le rôle et les enjeux de la conception spatiale dans ce contexte de forte mutation de la place de l'espace mis à la disposition du travail dans les entreprises.

101 Façonné par des codes symboliques domestiques (fauteuils, sofas, poufs, cuisine, etc.) et en n'étant qu'un lieu parmi d'autres, dans une constellation d'espaces de nature diverse, l'espace de l'entreprise change de statut. Il ne constitue plus la référence pour le travail qui peut s'opérer ailleurs. L'espace relative à la tâche se dissipe pour interférer et se confondre avec les espaces autres (urbains, privés ou sociaux).

102 En supprimant la contrainte de la distance physique, le numérique conduit à ne plus distinguer l'espace dédié à la tâche des autres lieux. L'espace à allouer à la production n'est plus une préoccupation nécessaire pour l'entreprise tertiaire, qui peut ne plus s'en soucier en offrant des édifices composés de plateaux offrant un panel de zones différenciées associées à la technologie numérique. Les espaces ne sont ainsi plus façonnés par le travail dans le temps long de l'expérience des employés qui désormais vivent une multiplicité des lieux en sein et en dehors de l'organisation du travail dans un rapport au temps plus ponctuel.

\subsection{Le registre conceptuel et méthodologique pour comprendre le travail}

Dans cette perspective d'évolution forte du numérique et de ses implications sur les espaces, quelques pistes peuvent être proposées pour saisir la question du travail sur le plan conceptuel et méthodologique.

\section{Interroger les méthodes pour prendre en compte les nouvelles formes d'articulation entre l'activité de travail et les espaces dans lesquelles elle prend place}

Dans une situation de forte porosité des espaces, interroger les nouvelles formes d'articulation entre l'espace et l'activité de travail dans des environnements multiples se pose. Le cadre pour analyser l'activité de travail dans un contexte de forte mobilité et de migration spatiale entre des sphères privées, publics et d'entreprise, se pose. Quel pont méthodologique construire pour analyser le travail réalisé dans l'entreprise, à domicile, dans les tiers lieux et autres espaces urbains? Comment caractériser ces espaces, leurs porosités et leurs imbrications ? Comment saisir la dimension mêlée du travail aux autres formes d'activités humaines : vie de famille et vie sociale dans un café, un bar ou un salon pour voyageurs?

Dans le déroulé de l'activité de travail, éclaté dans une diversité de lieux, quel est le rapport entre le temps et les espaces? Est-il possible d'envisager un rapport temporel plus long dans la manière dont le travail peut prendre place dans cette constellation d'espaces distincts ou est-ce qu'il est condamné à occuper ces espaces dans un temps court? Autrement dit, sommes-nous entrés dans une ère de simple occupation des espaces multiples et éparpillés sans les habiter dans la durée?

Comment étudier le travail dans ces environnements ne distinguant plus la présence physique des employés dans un lieu, des relations à distance avec des collaborateurs ailleurs, chez eux ou parfois à l'autre bout de la planète ? Comment se modèle ce milieu 
dans une approche réciproque intégrant le développement tant des activités que des espaces dans lesquels elles s'inscrivent?

107 Ces évolutions interpellent l'outillage méthodologique pratiqué en analyse de l'activité et historiquement relatif aux situations de travail dans les entreprises

\section{Revisiter la notion de situation de travail en l'enrichissant de la dimension spatiale}

108 La proposition développée ici est que ces formes éclatées du travail dans les espaces invitent à saisir simultanément la dimension spatiale et temporelle de l'activité de travail pour esquisser d'autres formes d'unité.

109 Elle propose de convoquer la notion de situation de travail développée en ergonomie en interrogeant sa déclinaison spatiale. L'analyse de l'activité, historiquement construite dans une unité spatiale et temporelle relative à l'entreprise, est aujourd'hui chahutée et invitée à évoluer pour prendre en compte cet éparpillement des espaces et des temps.

110 En enrichissant l'unité définie par la situation de travail, au travers d'une approche qui spatialise l'activité de travail, un cadre d'analyse peut être posé. Il vise à décoder la structure et l'organisation de cette fragmentation complexe et imbriquée d'espaces aux statuts distincts en lien avec l'activité de travail. L'accent est à mettre sur ce qui, dans la manière dont l'activité de travail délimite et remodèle cette constellation d'espaces qui lui sont propres, permet de dessiner les contours de la situation de travail.

111 Approcher spatialement la façon dont l'activité de travail agence et organise cette complexité spatiale et temporelle permet d'ébaucher les contours de la situation de travail. Une unité ayant une cohérence propre reliée à l'activité de travail dans un environnement qui fragmente les lieux de production au travers des technologies numériques.

112 La notion de situation de travail demeure selon nous l'unité pertinente pour délimiter le périmètre propre à la logique du travail humain imbriquant aujourd'hui tout aussi bien des espaces physiques et virtuels dans les modalités de régulation de l'activité individuelle et collective de production. Elle peut instruire comment se préfigure l'espace propre à l'activité dans le déroulé du travail à partir de repères imbriquant espaces physiques et espaces virtuels. Elle peut éclairer ces nouvelles logiques de déclinaison spatiale de l'activité construite à partir de repères dispersés et de nature différente (privé, public, professionnel).

113 Cet ensemble d'espaces de l'activité de travail trouve sa cohérence comme étant tout aussi bien le support que le produit de l'activité de travail. Si dans l'entreprise cet espace déborde déjà le découpage classique relatif à la tâche, comme cela a été évoqué plus haut, cet affranchissement des frontières est exacerbé avec l'introduction des technologies numériques combinée à ces nouveaux modèles d'espaces d'entreprises qui ne se préoccupent plus de l'espace dédié à la tâche.

114 La question des modalités méthodologiques pour saisir ces nouvelles logiques spatiales de l'activité de travail se pose, car elle nécessite de s'intéresser à des repères dispersés et de nature différente (privé, public, professionnel). Cet article ayant été réalisé dans l'enceinte d'une firme, l'analyse est maintenant à étendre au-delà de ce périmètre dans une approche globale d'une délimitation à dessiner par la situation de travail. La question des méthodes pour analyser le travail réalisé dans des sphères imbriquant dimensions privées, professionnelles, urbaines et sociales se pose. Elle reste à 
documenter, notamment en explorant les pratiques disciplinaires différentes pour engager une réflexion qui ne sépare plus le travail des autres dimensions de la vie.

\section{Conclusion et perspectives}

L'analyse de l'activité de travail réalisée dans le cas du modèle du flex office dans l'immobilier d'entreprise de cette firme du secteur tertiaire défend l'idée que la situation de travail est l'unité pertinent pour de délimiter l'espace qui émerge dans la dynamique de l'action du travail réel. La continuité est construite par l'activité de travail qui se déploie dans des espaces différents intra et extra entreprise et non pas par l'addition des espaces pensés dans le cadre du flex office au sein de l'organisation. Ces espaces configurés dans une logique de travail dans et en dehors de l'entreprise émergent de l'activité de travail. Pour celui ou celle qui réalise le travail, cet ensemble est la référence concrète, elle est partiellement matérielle et partiellement immatérielle au travers des rapports et des relations construits par et dans le travail.

116 Une distinction est en effet à opérer entre lieu et espace. Le flex produit des espaces homogènes et non des lieux. Un lieu est singulier et concret (de Certeau, 1990). Il mêle action de travailler, pratiques narratives, représentation et configuration spatiale. Il a un sens et est intelligible pour celles et ceux qui le pratiquent de manière singulière (Lautier, 1996). La standardisation de l'espace physique, tout en l'étendant au travers des relations induites par les technologies, conduit à un espace sans limites. L'introduction massive du numérique conduit à non seulement supprimer la contrainte matérielle de la distance, mais aussi à ne plus spécifier l'espace dédié au travail. Peu importe où le travail se passe. Cette rationalité de l'espace, conçue à partir d'une organisation qui place au centre, les nouvelles technologies numériques pour relier et produire, bouscule les limites classiques de l'espace de production.

117 Cette dynamique spatiale et temporelle de l'activité de travail qui déborde l'unité historique du travail en entreprise, interroge le devenir des lieux de travail dans un contexte où l'espace de l'entreprise n'est plus la référence pour le travail. Ce faisant, elle questionne les interfaces entre les "espaces / temps" privés, sociaux et professionnels. La recherche d'un équilibre et d'une cohérence d'ensemble semble être laissée à la charge des employés qui, d'une certaine manière, déportent la dimension professionnelle en dehors de l'entreprise dans une constellation complexe de lieux différenciés, au risque d'une perte de repères. En explorant différentes pratiques d'espaces, ils peuvent construire dans le même temps, de nouvelles formes d'unités spatiales à partir de la logique de l'activité de travail.

118 Pour terminer, cela a été évoqué, les résultats développés dans cet article remontent à 2019. Même s'ils peuvent faire écho à l'actualité de la situation induite par la crise sanitaire avec la généralisation du travail en dehors des l'entreprise au travers du télétravail, une prudence méthodologique s'impose. Poursuivre cette réflexion pour prendre la mesure de ces nouvelles réalités du travail contemporain, y compris sur le plan spatial, est un axe de recherche à développer. 


\section{BIBLIOGRAPHIE}

Berque, A. (2009). ÉCOUMENE. Introduction à l'étude des milieux humains. Paris: Éditions Belin.

Camus, C., Évette, T., \& Fabre, A. (1991). La conception des lieux de travail. Une ressource pour l'entreprise. Montrouge: Éditions de l'ANACT, collection points de repère.

Canguilhem, G. (1943). Le normal et le pathologique. Paris: Presses Universitaires de France.

Cazamian, P. (1996). À la recherche d'une science globale de l'homme. In P. Cazamian, F. Hubault, \& M. Noulin. (Eds.), Traité d'ergonomie. Nouvelle édition actualisée (pp. 461-464). Toulouse: Octarès.

Cazamian, P., \& Lautier, F. (1996). Les concepts de temps et d'espace. Leur évolution historique. In P. Cazamian, F. Hubault, \& M. Noulin. (Eds.), Traité d'ergonomie. Nouvelle édition actualisée (pp. 461-464). Toulouse: Octarès.

Certeau (de), M. (1990). L'invention du quotidien. I. Arts de faire. Paris: Gallimard.

Couillaud, S. (2018). Développement du pouvoir d'agir des acteurs en co-innovation par les usages : l'exemple d'accompagnement du projet d'habitat évolutif pour séniors. [Thèse de doctorat en ergonomie]. Université Paris 8.

Daniellou, F. (1988). Ergonomie et démarche de conception dans les industries de process continus, quelques étapes clefs. Le Travail Humain, 51(2), 184-194.

Daniellou, F. (2004). L'ergonomie dans la conduite de projets de conception de systèmes de travail. In P. Falzon (Ed.), Ergonomie (pp. 359-373). Paris: PUF.

Daniellou, F., \& Garrigou, A. (1993). La mise en œuvre des situations passées et des situations futures dans la participation des opérateurs à la conception. In A. Weill-Fassina, P. Rabardel, \& D. Dubois (Eds.), Représentations pour l'action (pp. 359-374), Toulouse: Octarès.

Dejean, P.H., Pretto, J., \& Renouard, J.P. (1988). Organiser et concevoir les espaces de travail. Montrouge: Anact.

Falzon, P. (2013). Pour une ergonomie constructive. In P. Falzon (Ed.), Ergonomie constructive (pp. 1-15). Paris: PUF.

Fenker, M. (2003). L'espace : un mode de gestion de la dynamique organisationnelle. [Thèse de doctorat en sciences de gestion]. École polytechnique.

Fischer, G-N. (1978). L'espace comme nouvelle lecture du travail. In Sociologie du travail, $20^{\mathrm{e}}$ année n4, octobre-décembre 1987 (pp. 397-422) Aux éditions du seuil. DOI : https://doi.org/10.3406/ sotra. 1978.1590

Fischer, G-N. (2016). Les espaces de Travail. Enjeux Humains. In F. Dortier (Ed.), La Communication. Des relations interpersonnelles aux réseaux sociaux (pp. 168-178). Éditions Sciences Humaines.

Gadrey, J., \& Zarifian, P. (2003). L'émergence d'un modèle du service : enjeux et réalités. Paris: Éditions Liaisons.

Gaudart, C. (2014). Les relations entre l'âge et le travail comme problème temporel. Perspectives interdisciplinaires sur le travail et la santé [En ligne], 16(1) URL : http://journals.openedition.org/ pistes/3052 ; DOI : https://doi.org/10.4000/pistes.3052

Goffman, E. (1973). La mise en scène de la vie quotidienne. Paris: Éditions de Minuit. 
Goffman, E. (1974). Les rites d'interaction. Paris: Éditions de Minuit.

Hall, E. T. (1966/1971). La dimension cachée. Paris: Points seuil.

Heddad, N. (2017a). L'espace de l'activité : Une construction conjointe de l'activité et de l'espace. Le Travail Humain, 80(2), 207-233. DOI 10.3917/th.802.0208.

Heddad, N. (2017b). Intervenir pour la conception de l'espace de l'activité : vers un cadre méthodologique. Activités [En ligne], 14(1). DOI : https://doi.org/10.4000/activites.2958

Kant, E. (1968/1781). Critique de la raison pure (trad. A. Tremesaygues et B. Pacaud). Paris: PUF.

Kirsh, D. (1995). The intelligent use of space. Artificial Intelligence, 773, 31-68.

Lautier, F. (1996). Espaces et organisation. In P. Cazamian, F. Hubault, \& M. Noulin. (Eds.), Traité d'ergonomie. Nouvelle édition actualisée (pp. 492-507). Toulouse: Octarès.

Lautier, F. (1999). Ergotopiques. Sur les espaces des lieux de travail. Toulouse: Octarès.

Lautier, F. (2001). Penser l('espace d)e travail. In F. Hubault (Ed.), Comprendre que travailler c'est penser, un enjeu industriel de l'intervention ergonomique (pp. 109-119). Toulouse: Octarès.

Lefebvre, H. (1974/ 2000). La production de l'espace. Paris: Anthropos.

Martin, C. (2000). Maîtrise d'ouvrage, maîtrise d'œuvre : construire un vrai dialogue. La contribution de l'ergonome à la conduite de projet architectural. Toulouse: Éditions Octarès.

Merleau-Ponty, M. (1945). Phénoménologie de la perception. Paris: Gallimard.

Poincaré, H. (1902, 1968, 2014). La science et l'hypothèse. Paris: Clamps-Flamarion.

Ricœur, P. (1969). Le conflit des interprétations, Essais d'herméneutiques. Paris: Éditions du Seuil.

Simondon, G. (1958). Du mode d'existence des objets techniques (1989). Paris: Aubier.

Suchman, L. (1996). Constituting shared workspaces. In Y. Engeström, \& D. Middleton (Eds.), Cognition and communication at work (pp. 35-60). New-York: Cambridge University Press.

Varela, F., Thompson E., \& Rosch E. (1993). L'inscription corporelle de l'esprit. Sciences cognitives et expérience humaine. Paris: Éditions du Seuil.

Zetlaoui-Léger, J. (2013). Urbanisme participative. In I. Assilo, R. Barbier, L. Blondiaux, F. Chateauraynaud, J.M. Fourniau, R. Lefebvre, C. Neveu, \& D. Salles (Eds.). Dictionnaire critique et interdisciplinaire de la participation (pp. 2268-5863). Paris: Groupement d'Intérêt Scientifique Participation du public, décision, démocratie participative.

Zouinar, M. (2020). Évolutions de l'Intelligence Artificielle : quels enjeux pour l'activité humaine et la relation Humain-Machine au travail ? Activités [En ligne], 17(1) mis en ligne le 15 avril 2020. URL : http://journals.openedition.org/activites/4941 ; DOI : https://doi.org/10.4000/activites.494

\section{NOTES}

1. Une distance rapprochée entre deux interlocuteurs est par exemple, vécue comme une intrusion en Scandinavie, alors qu'elle est une condition pour un échange pour des personnes des pays du sud. Cette idée peut se retrouver dans les entreprises. Selon le lien hiérarchique, les personnes ne se tiennent pas à la même distance pour échanger. La distance est généralement plus grande entre un responsable et un agent qu'entre collègues d'un collectif de travail.

2. La figure 1 montre un exemple d'un opérateur installé à un poste dans un local. Les deux sont des moyens spatiaux fournis par l'organisation du travail. La figure 2 montre un exemple où le 
déplacement du poste de travail est opéré par l'agent de façon à lui permettre un visuel sur une collègue de travail, sur la porte d'accès et sur un tableau d'indicateurs disposé sur un mur. L'agent transforme ici la disposition spatiale du poste et du local qui lui sont attribués. Les relations auxquelles l'espace peut répondre sont aussi bien de l'ordre visuel, mais aussi sonore (pour entendre) ou même pour sentir (courant d'air, odeur, etc.).

3. Il faut signaler que cet article a été rédigé à l'issue d'une journée thématique sur la question de l'espace et le numérique réalisée en mai 2019, c'est-à-dire avant la période de pandémie qui a depuis modifié la situation. Les données sont issues d'une analyse réalisée à une époque où le modèle spatial du flex office (bureau flexible) constituait une offre nouvelle mise en valeur dans l'immobilier tertiaire en France.

4. Les entretiens et les observations ont été organisés la façon suivante :

- Des entretiens individuels ont été conduits avec les responsables, mais aussi avec des collaborateurs identifiés comme réalisant une activité pour une grande partie individuelle.

- Des entretiens collectifs ont été menés avec des salariés réalisant collectivement l'activité de travail.

- Des observations ont été réalisées en situation réelle d'activité sur la base d'une sélection de 3 catégories de journées significatives: lors des jours du mois dédiés aux réunions de service caractérisées par la présence de tous les collaborateurs dans les lieux; lors des jours de la semaine marqués par une présence d'un nombre significatif des collaborateurs et lors des jours de la semaine avec une faible présence des salariés dans les locaux. Les observations ont été réalisées en continu sur la journée de travail.

5. Le projet d'aménagement distingue ainsi les catégories suivantes: (1) espace "silence / concentration", (2) espace "nomade silence», (3) espace de travail traditionnel « concentration", (4) espaces "projet : travail débout sur un bench haut», (5) espaces " projet pour un travail en posture assise sur des bench bas" (6) espace de «travail silencieux" (7) espace «isoloir téléphone », (8) espace «isoloir à deux », (9) espace de "convivialité / idéation », (10) espace de réunion et (11) espace de détente commun.

6. Par exemple, l'espace "silencieux / concentration » est encloisonné, légèrement à l'écart et aménagé avec des postes en posture assise haute ou basse sur des plans de travail en longueur. L'espace "projet» est ouvert, situé dans une zone centrale et composé de bench ou tables rondes. L'espace « nomade silence » est agencé en alcôve légèrement en retrait.

7. Dans un des espaces "projet » équipés de benchs hauts ou de postes classiques, dans l'un des espaces « isoloir à deux », ou dans l'un des espaces de " convivialité / idéation », notamment un coin de la cuisine ou encore dans l'un des salons, ou dans une salle de réunion de l'étage ou ailleurs dans l'immeuble ou encore à l'extérieur de l'entreprise?

8. Le catalogue des mobiliers et le cahier des tendances sont proposés et portés par des cabinets de conseil en design et aménagement et sont bien souvent proposés de la même façon d'une entreprise à l'autre.

\section{RÉSUMÉS}

Cet article s'ancre dans le champ de l'ergonomie de l'activité. Il propose d'aborder la question spatiale dans les organisations de travail autour du modèle du flex office. Une première partie traite de la spécificité des espaces dédiés au travail. La question de la subordination du travail les distingue en effet clairement des autres lieux de la vie. Une deuxième partie propose une 
approche théorique de l'espace pour poser un cadre visant à saisir dans un même temps, la question de l'activité de travail et celle de l'espace. L'espace pensé pour la tâche se différencie de l'espace modelé et agi par l'activité de travail. Une troisième partie aborde la question du lien observé entre le digital et l'évolution des espaces dans l'immobilier des entreprises avec l'introduction du modèle du flex office dans le tertiaire. Une quatrième partie présente l'étude de cas et les méthodes utilisées dans le cadre d'un retour d'expérience. Une cinquième partie met l'accent sur les caractéristiques des espaces dans le modèle du flex office. D'un côté, elles questionnent la manière dont l'espace, conçu par les concepteurs, participe de la tâche et de l'autre, la manière dont l'activité de travail s'y déploie. Le travail se réalise dans une porosité spatio-temporelle dans et hors de l'entreprise. Ce faisant, l'activité de travail en flex office contribue à renforcer le développement du numérique, à la fois condition et résultante du travail en flex office. Enfin, une dernière partie ouvre sur la discussion et les perspectives du point de vue du travail

This article aims to address the issue of space in work organization in the context of the development of digital technology and space. We offer an insight into flex offices as a new model of workspace. This paper is anchored in the field of the ergonomics with a focus on activity. We describe the link between work activity and space. The paper is organized into six parts. The first part deals with the specificity of space in work organizations. The relation to subordination is singular and linked to work in companies. It is possible to distinguish between spaces dedicated to work and other types of space. Working space is different from housing or urban space. The second part proposes a theoretical approach to space as a framework to simultaneously reach work activity and its space. The space designed by the organization or designers (the space of the task) differs from the space modeled by the work activity (the space of the activity). The third part examines the evolution of digital technology in the context of spaces dedicated to work. A link is observed between the development of digital technology and the evolution of space in companies. The fourth part presents the case study and the methods used to provide feedback after installation in a flex office. The fifth part develops the main results of the analysis by focusing on the characteristics of the spaces in the flex office model. The first two characteristics focus on how space, designed by designers, participates in the task. The last two characteristics show how the work activity unfolds and takes place in new spaces. The sixth and final part opens up perspectives from a work perspective.

INDEX

Keywords : work, design, flex office, digital technologies and activity's space

Mots-clés : travail, conception, flex office, technologie numérique et espace de l'activité

\section{AUTEUR}

\section{NADIA HEDDAD}

Pôle Ergonomie. FCPS, Université Paris 1. Panthéon Sorbonne. 21 Rue Broca 75005 Paris nadia.heddad@icloud.com 